\title{
AGORA: An Integrated Knowledge Management Environment
}

\author{
P.M. Chrissohoos, M.P. Anastasiou, I.P. Kouranos, N.N. Kalogeropoulou, \\ M.P. Aslani \\ INTRACOM S.A.,Greece \\ Em: phry@intracom.gr
}

Keywords Knowledge management, Knowledge awareness, GLOBEMEN

\begin{abstract}
Telecommunication Industries in their efforts to ensure long-term survival in today's rapidly changing business environment, they should increase their responsiveness and be both pro-active and re-active to challenges driven from their environment. In an effort to address this issue, INTRACOM will demonstrate the use of an integrated knowledge management environment called "AGORA" aiming at increasing its responsiveness of the Sales and Marketing business process and support the execution of this process in an inter-company environment.
\end{abstract}

\section{INTRODUCTION}

In today's global business environment, telecommunications industries face new challenges. Market liberalisation, privatisation, deregulation, and the rapid deployment of new information and communication technologies have lead to a business environment that changes often, irregularly and almost unpredictably. In order to ensure long-term survival, telecommunications industries should develop and deploy new services rapidly, in order to differentiate themselves from their competitors. Key factors of differentiation are the external and internal responsiveness, and knowledge awareness. The external awareness of an enterprise refers to its ability to understand its external environment, while its internal awareness is the enterprise's ability to understand itself, its strengths and weaknesses [1]. Being aware of its competencies and skills does not provide the enterprise with a clear path to successful performance, unless it is combined with its ability to efficiently manage internal change and leverage internal 
competencies and resources to meet a market opportunity or respond to a customer need [2]. This enterprise ability is called internal responsiveness [2]. In that way the enterprise is able to rapidly and efficiently respond to challenges driven from the external environment and outplay its competitors [1].

In this context, knowledge management is becoming of increasing importance to industry and organisations, in order to improve organisational efficiency, competency and innovation. Knowledge management involves the identification and analysis of available and required knowledge assets and their knowledge related processes, as well as the subsequent planning and control of actions to develop both the assets and the processes so as to fulfill organisational objectives [3].

\section{OBJECTIVES}

In its effort to address the above mentioned issues, INTRACOM, the largest Hellenic industrial group in the field of Telecommunications, Electronics and Information Technologies, aims at increasing the responsiveness of the Sales and Marketing business process, so as to be able to rapidly and successfully respond to global market opportunities, and to achieve what is called "global reach".

From this perspective, the above-mentioned objective could only be achieved through the company's acquisition of knowledge awareness of the Sales and Marketing process. This business process is a very complex and critical one, and its main activities are:

- Selection, analysis and refinement of information related to market trends, market place competition, identified opportunities and potential problems in the marketplace.

- Monitoring of the technology advancements, aiming at identifying key opportunities of technological growth.

- Identification, contact establishment and acquisition of new customers.

- Identification of customers' requirements for product enhancement and/or new products and services development.

- Management of the existing company's business network, aiming at ensuring its responsiveness to future market opportunities. This business network consists of the company's partners including suppliers, contractors, sub-contractors and engineering offices, which may all be spread over different sites and/or geographical regions.

- Response to Request for Tenders by preparing a bidding document in compliance with the Tendering organisation's criteria and requirements. 
The efficient performance of the above activities presupposes the effective management of a large amount of information and knowledge. Despite that, there is an inherent complexity in managing this knowledge that stems from the fact that various relationships and dependencies exist between the various knowledge categories. Moreover, this knowledge is globally distributed in different company departments, as well as in different organisations within the business network, thus creating communication difficulties and sharing inefficiencies. It is often the case that the required knowledge resides in many different places and sources, such as the WEB, press releases and existing databases in the business network. In addition, the individuals involved in the process have important process - relevant knowledge that could be helpful to their colleagues if it could be derived and be available to them in some way.

Therefore, INTRACOM aims to implement a prototype of the "AGORA" and demonstrate its use as an integrated knowledge management environment. This prototype will be implemented throughout INTRACOM's involvement in the IMS project "GLOBEMEN" (Global Engineering and Manufacturing in Enterprise Networks), aiming at supporting and enhancing the performance of the Sales and Marketing inter-enterprise business process.

\section{THE AGORA ENVIRONMENT}

AGORA environment will consist of practices and applications that will enable the efficient acquisition, use, organisation and transfer of globally distributed implicit and explicit knowledge, related to the Sales and Marketing process.

\subsection{AGORA modules}

"AGORA" currently undergoes the phases of requirements and functional specification definition. The study of the Sales and Marketing process performed until this stage, indicated that "AGORA" should provide the process actors with timely access to valuable knowledge at anytime and anywhere in a fast and secure way. Such being the case, AGORA will operate over the INTERNET providing all users with access to the required knowledge via their web browser.

Based on the preliminary requirements derived until this stage, AGORA should incorporate the following four basic modules:

- A Knowledge Repository. This repository will be populated with information and knowledge related to the Sales and Marketing process. 
The development of the repository should cater for the efficient management of its content, as well as for security issues. Furthermore, the user will be provided with efficient support to access and update the required information/knowledge.

- The Knowledge Explorer module. Since the information required to Sales and Marketing process is subject to irregular and unpredictable change, this module will provide the process participants with the required functionality to search for valuable information in various sources, such as WEB, local drives and CDs. The user will be able to select the most valuable parts of the information found and store it in the knowledge repository.

- The Knowledge Organiser module. This module will cater for rolebased and personalised presentation of the available information and knowledge. This means that the users will be able to quickly and easily obtain the information and knowledge that is relevant to their task and responsibilities. Moreover, the users will be facilitated to organise existing knowledge and information, as well as to view it from different perspectives, depending on their role in the process.

- The Knowledge Report Generator module. This module will provide the actor of the Sales and Marketing process with functionality to aggregate existing knowledge, synthesise different pieces of information and generate reports on process related issues. An indicative list of such reports is: technology trends, existing and new products or services, market trends in a specific region. 


\subsection{Technical Solutions}

The development of the AGORA environment will exploit productive Internet technologies and tools that support the rapid design, refinement, extension and integration of the various applications. In the following paragraphs the selected technical solutions are briefly described.

- The 3-tier Architecture. The three-tier architecture platform is today the dominating Internet platform. Software vendors, such as Oracle, IBM, HP and Sun are using it, in order to provide a business framework that Internet applications can rely on. This architecture consists of three tiers. The Web Server, the Application Server, and the Database Server (Figure 1).

The Web Server "listens" requests made up by web browsers through the World Wide Web. A client's request goes to the Web Server, where authentication issues and problems are solved. This request is then passed to the Application Server, which actually manages all the requests that the Web Server passes in.

The Application Server as a management console allocates time and space, in order to successfully let the client execute the application making use of the data stored in the Database Server. The whole platform runs with accuracy, enabling safe, complex and even parallel transactions to be completed fast.

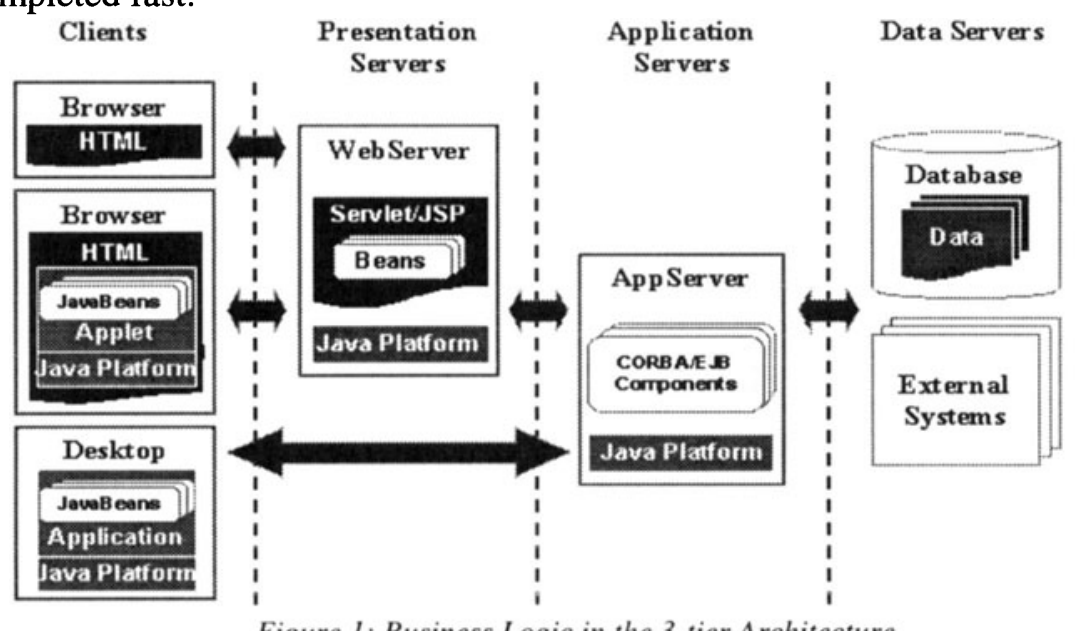

Figure 1: Business Logic in the 3-tier Architecture

The above architecture makes use of state-of-the-art technologies:

- Java. The Java programming language is the language of Internet. New programming features have been proposed by Sun, such as Java Server Pages (JSP), Enterprise Java Beans (EJBs), and Java Servlets, which are used by many vendors in their platforms to achieve what is called business 
logic. The biggest advantage by using these programming techniques is that a Java-enabled browser can run such an application with no additional requirements (space, memory, add-ins). Moreover, the Java language provides a very strong framework in which security and communication issues are being addressed.

- XML. The eXtensive Markup Language (XML) provides capabilities of efficient data exchange, in cases where the content of the information can be stored and further retrieved with ease and accuracy. The HTML language is the best language known so far for the presentation of information and data that resides in a RDBMS system to an Internet site. However, it could not address problems where the content and the information itself is so valuable and critical that need to be stored with accuracy, and further retrieved or updated if needed. XML can now address these problems [4,5]. Furthermore, Java provides the framework to parse, change, exchange and even store that information in a RDBMS system.

- Oracle. Oracle 8.i was the first xml-enabled database. With many capabilities such as backup and recovery, Oracle can be customised and optimised according to user needs, providing a very accurate, powerful and user friendly RDBMS system. It works ideally with Java (JDBC drivers, SQLJ) and even its own language (PL/SQL) can be used to obtain the best results.

The implementation of the "AGORA" environment will be based on this 3-tier architecture, consisting of a collection of Enterprise Java Beans, Java Servlets and Java Server Pages, each of which will play its own role towards the application development. Java APIs will be used to transform XML documents into valid ones (capable to be stored in the RDBMS). The RDBMS system will be Oracle 8.i. Finally, the choice of the Application Server would be based upon real-time results that would be obtained when AGORA's environment will be published over the Web.

\section{ANTICIPATED USAGE OF AGORA ENVIRONMENT}

AGORA will allow enterprise wide collection, organisation, navigation, searching and dissemination of knowledge and information that are required by Sales and Marketing process owners, in order to perform their every day activities tasks.

Potential users of AGORA environment are personnel from the Sales and Marketing headquarters, sales representatives in a region, regional partners, and R\&D personnel.

Sales representatives will be responsible for enriching the content of the knowledge repository with information and knowledge about: 
- Market trends in the region of their responsibility

- Potential new customers in the region

- Customers requirements either for new products / services or for product enhancements

- Anticipated upcoming requests for tenders

- Competitors activities in the region

An operational scenario of the anticipated usage of AGORA environment is depicted in the above figure - Figure 2.

Regional partners will have access to the Knowledge Repository according to their role in the Business Network and in that way they will be able to find in it information and knowledge concerning their region and activities of the Business Network. In addition, they will be responsible to update the Knowledge Repository with their new R\&D plans, and possible activities in the region.

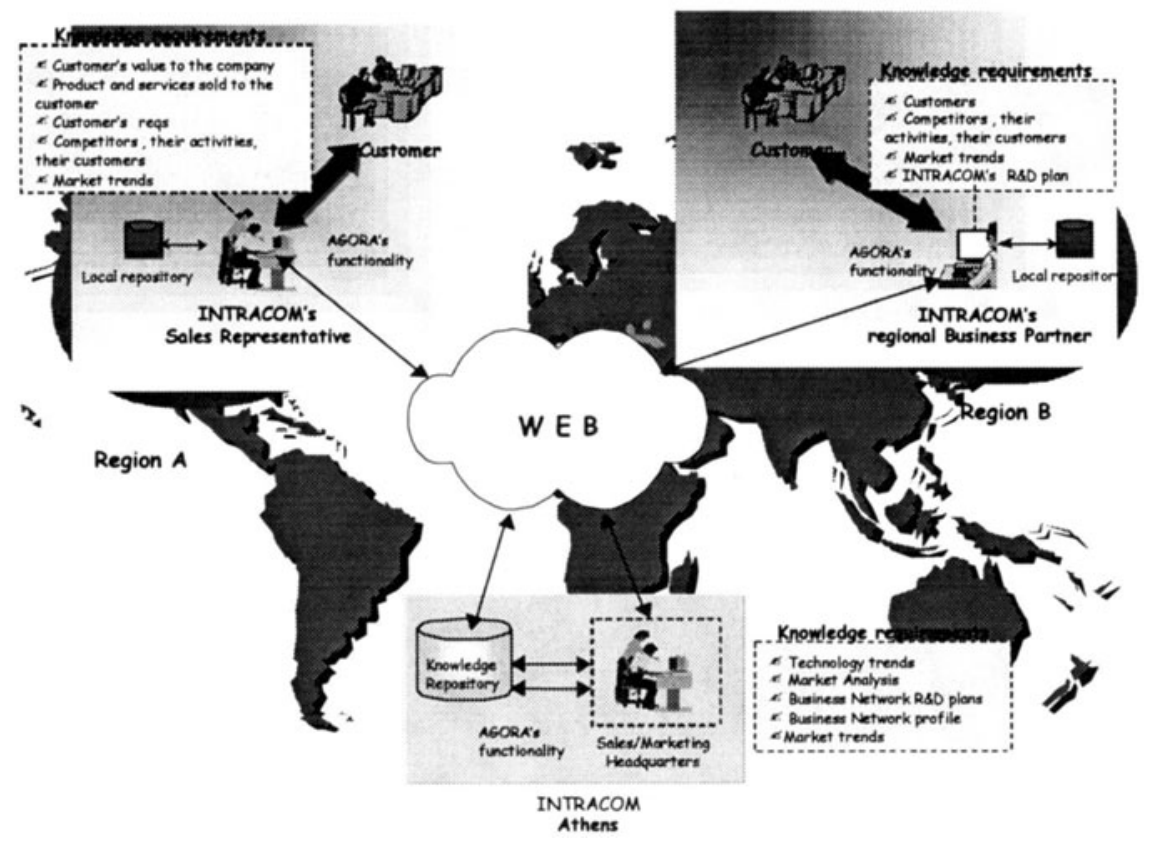

Figure 2. Anticipated usage of AGORA environment

R\&D personnel use the environment to search for information about emerging technologies and will have to enrich the content of the knowledge repository with reports on technology innovation and key opportunities of technological growth.

Personnel from Sales and Marketing headquarters will examine the knowledge generated from the above mentioned actors, as well as 
information about existing competencies in the Business Network, in order to define product and market strategic plans, R\&D plans and requirements for new business alliances.

Furthermore, it is anticipated that the use of AGORA for fulfilling Sales and Marketing actors' information and knowledge requirements will cultivate working practices that promote knowledge sharing and as a result continuous organisational learning. In that way, it is anticipated that in the long term AGORA will facilitate the development of the internal and external knowledge awareness and responsiveness of both INTRACOM and it business partners.

\section{CONCLUSION}

This paper presents through the "AGORA" environment, INTRACOM's approach and practice towards the management of knowledge related to the Sales and Marketing inter-enterprise business process. Throughout INTRACOM's involvement in the GLOBEMEN project, it is anticipated that a prototype of the "AGORA" will be developed and demonstrated at its pilot site. . The preliminary requirements that the AGORA environment should fulfil in an inter-enterprise environment have been currently derived. Moreover, a study of the state-of-the-art development tools and technologies was performed, and the most appropriate ones for the development of AGORA were selected. Future work will be focused on implementing a prototype of the "AGORA" environment and demonstrating its practical use in a real case scenario. Moreover, during the following project phases, methods of using such environment will be determined, in order to efficiently manage Sales and Marketing knowledge. In that way new working practices will be defined and modeled, which will promote learning and knowledge management sharing in a virtual enterprise network.

\section{REFERENCES}

[1] Thomas Koulopoulos, The Delphi Group, (1997). Creating Corporate Instinct, BusinessWeek

[2] Carl Frappaolo, What's Your Knowledge IQ?, ", Intelligent KM, http://www.intelligent km.com/feature/08/feat $1 . \mathrm{shtml}$

[3] Yogesh Malhotra (1998). Knowledge management for the New World of Business, @Brint.com, http://www.brint.com $/ \mathrm{km} /$ whatis.htm

[4] Magnus Lonnroth, (1999). Tech Brief: Introduction to XML, Oracle Corporation, http://www.oracle.com/oramag/webcolumns/TB03xm101.html

[5] Rohib Khare, Adam Rifkin (1997). Capturing the State of Distributed Systems with XML, World Wide Web Journal Special Issue on XML, 2, 207-218 